Relations industrielles

Industrial Relations

\title{
Législation du travail
}

\section{Les arbitres ont-ils juridiction pour décider des moyens de droit présentés au cours de l'arbitrage par l'une des parties?}

\section{Jean Gagné}

Volume 4, numéro 2, octobre 1948

URI : https://id.erudit.org/iderudit/1023436ar

DOI : https://doi.org/10.7202/1023436ar

Aller au sommaire du numéro

\section{Éditeur(s)}

Département des relations industrielles de l’Université Laval

\section{ISSN}

0034-379X (imprimé)

1703-8138 (numérique)

Découvrir la revue

Citer cet article

Gagné, J. (1948). Législation du travail : les arbitres ont-ils juridiction pour décider des moyens de droit présentés au cours de l'arbitrage par l'une des parties ? Relations industrielles / Industrial Relations, 4(2), 20-20.

https://doi.org/10.7202/1023436ar

Tous droits réservés (C Département des relations industrielles de l’Université Laval, 1948
Ce document est protégé par la loi sur le droit d'auteur. L'utilisation des services d'Érudit (y compris la reproduction) est assujettie à sa politique d'utilisation que vous pouvez consulter en ligne.

https://apropos.erudit.org/fr/usagers/politique-dutilisation/ 


\section{Législation du travail}

\section{Les arbitres ont-ils juridiction pour décider des moyens de droit présentés au cours de l'arbitrage par l'une des parties?}

Dans une brochure sur la \&Législation du travail >, extraite de la Revue du Barreau (février 1948) ${ }^{1}$, Me MarieLouis Beaulieu, avocat au Barreau de Québec, se propose de faire quelques réflexions sur la juridiction des arbitres en matière de droit, la reconnaissance syndicale et le fonctionnement de notre système double de convention collective, soit la convention particulière et la convention extensionnée, réflexions suggérées par un cas-type d'arbitrage où il agissait lui-même comme arbitre.

Ce litige est un différend survenu entre l'Union des Ouvriers de Salaison d'Amérique et l'Association des marchands détaillants du Canada Inc. (section de Québec), à l'occasion de la négociation d'une convention collective destinée à demeurer un contrat collectif particulier.

Me Beaulieu analyse spécialement l'aspect légal du problème suivant: les arbitres ont-ils juridiction pour décider des moyens de droit présentés au cours de l'arbitrage par l'une des parties, en l'occurrence l'Association des marchands détaillants?

L'auteur admet d'abord, en accord avec la jurisprudence existante, \& qu'il n'appartient pas aux arbitres * de se prononcer sur la question de savoir si le litige dont ils sont saisis pouvait leur être soumis.

Cela signifierait-il que les arbitres ne peuvent décider des questions de droit, s'ils en ont la compétence? Il ajoute que tel n'est pas le cas. Et c'est à l'étude de cet aspect de la question que Me Beaulieu s'applique ensuite.

Il base son argumentation sur le fait que notre Loi des différends ouvriers de Québec (1901), qui contient ce qui a trait à la composition des conseils d'arbitrage et à la nomination de ses membres, est basée sur une loi de la Nouvelle-Zélande (The Industrial Conciliation and Arbitration Act). Or dans ce pays, la Commission d'Arbitrage, se basant sur un article de cette loi où l'on parle « d'équité et de bonne conscience », a toujours décidé les questions de droit. Il poursuit en disant que dans la province de Québec, alors que les mêmes mots sont inclus dans notre Loi des différends ouvriers de Québec (a. 24) la question est de savoir si, pour dire que les arbitres ne peuvent décider les problèmes de droit quand ils en ont la compétence, on peut invoquer l'obligation qui leur incombe de décider le différend \&suivant l'équité et la bonne conscience».

Me Beaulieu affirme alors que «c'est en décidant, in equity and good conscience, que la Commission d'Arbitrage juge des litiges qui lui sont soumis, cela, tant sous leurs aspects légaux que sur les faits, les problèmes économiques, professionnels ou autres $\gg$.

Pour répondre à ceux qui interprètent le texte examiné comme imposant aux arbitres lobligation de décider les différends en faisant abstraction des lois », Me Beaulieu est amené à étudier ce qu'on entend aujourd'hui en droit anglais ( vu l'origine de notre loi), par le mot $<$ equity $>$.

Dans un rapide retour sur le passé, il fait l'historique de l'apparition de l'équité dans le droit anglais, de l'évolution de son application dans le système juridique anglais jusqu'à nos jours, et de l'importance grandissante

(1) La brochure est en vente chez l'auteur, 111, côte de la Montagne, Québec qu'elle y a prise. Il dit, en s'inspirant d'une citation de Lord Talbot, que \&l'équité est une vertu morale qui qualifie, tempère et réforme la rigueur, la sévérité et le tranchant de la loi. Elle prête assistance à la loi, là où cette dernière est faible et défectueuse, et la défend contre les subterfuges, les erreurs et les subtilités qui servent souvent à priver de remède ceux qui ont des droits incontestables. C'est là la fonction de l'équité, qui assiste et protège le common law. L'équité, par conséquent, ne détruit pas la loi, non plus qu'elle lui donne naissance, mais l'assiste $\gg$.

Me Beaulieu conclut qu'il n'y a pas que les aspects légaux du différend que les arbitres doivent décider \& suivant l'équité et la bonne conscience ». C'est tout le différend. Le texte s'applique donc d'après lui aux questions de droit comme aux questions de fait, aux problèmes d'ordre économique, professionnel, etc., tels que les salaires, les heures de travail, les congés, la sécurité syndicale.

Me Beaulieu ne se contente pas de cet exposé. Il invoque encore, à l'appui de sa thèse, la Loi des relations ouvrières et la Loi des différends entre les services publics et leurs salariés.

Il rappelle alors l'interprétation de l'article 4 de cette dernière loi que deux bancs de la Cour d'Appel viennent de donner, dans la cause de l'Association catholique des institutrices du district no 16 Inc. vs Les Commissaires d'écoles pour la Municipalité de St-Athanase "2. Me Beaulieu traite de cette cause et en relève les points légaux les plus intéressants. L'auteur cite à profusion les notes de Phonorable Juge Pratte, reconnu comme une autorité en la matière.

A la lumière de tous les principes évoqués dans ces considérations, Me Beaulieu analyse ensuite le différend qu'il s'est proposé d'étudier au début de son étude. Il reclierche quel est l'effet de la reconnaissance syndicale dans le cas précité. Il dit que, dans le cas, \&'obligation de négocier incombe uniquement à l'Association (des marchands détaillants), et non aux employeurs eux-mêmes, nais cette obligation, dit-il, peut-être différente pour chacun de ses membres, elle peut même exister pour tel ou tel membre et non pour tel ou tel autre. Ainsi, l'Association n'est pas obligée de négocier pour ses membres dont les salariés n'ont pas de reconnaissance syndicale s. Cette constatation très intéressante nous apporte un point de vue nouveau dans l'interprétation de nos lois du travail sur le sujet.

La lecture de cette étude devrait être de grande utilité pour tous ceux qui s'intéressent à la législation du travail, parce que, comme nous l'avons déjâ souligné, l'auteur y présente des remarques de haute valeur sur le conseil d'arbitrage, le rôle des arbitres en matière de droit, l'équité et la bonne conscience dans l'interprétation de nos lois du travail, la reconnaissance syndicale, la convention collective et les distinctions à faire entre la convention collective particulière et le décret.

Nous aimerions pouvoir lire plus souvent les exposés méthodiques et lumineux de cet auteur sur les importants problèmes légaux que soulèvent les relations du travail dans notre province.

Jean Gagné 